\title{
Hopf Bifurcation and Stability Analysis for A Delayed Logistic Equation
}

\author{
F. Bozkurt
}

\begin{abstract}
This paper deals with the stability analysis and theHopf bifurcation at the equilibrium points of a logistic delay differential equation.By applying the Halanay inequality, the local stability of the logistic differential equation is discussed. The stability of bifurcation periodic solutions and the direction of Hopf bifurcation are determined by applying the normal form theory and the center manifold theorem. Numerical examples show interesting nonlinear behavior of the logistic differential equation at the end of the paper.
\end{abstract}

Index Terms-Differential equations with delay, Hopf bifurcation, local stability, global asymptotic stability, periodic solutions.

\section{INTRODUCTION}

Researches about the delayed logistic equations have received significant attention in the recent years [1]-[6], especially from biologists and mathematicians. Hutchinson [1] assumed egg formation to occur $\tau$ units of time before hatching and proposed the following delayed logistic equation:

$$
\frac{d x}{d t}=r \cdot x(t)\left\{1-\frac{x(t-\tau)}{K}\right\}
$$

where $r>0$ is intrinsic growth rate, $K>0$ is the carrying capacity of population and $\tau>0$ is time delay.Properties of Eq. (1.1) were studied by various authors [7]-[9]. It was shown in [4] that for parameters $r$ and $\tau$ the equilibrium point $x=K$ is locally asymptotically stable.

K. Gopalsamy [2] considered a realistic and general case of Hutchinson's equation

$$
\frac{d x(t)}{d t}=r \cdot x(t)\left\{1-a_{1} \cdot x(t)-a_{2} x(t-\tau)\right\}
$$

where $a_{1}, a_{2}$ and $r$ are positive constants. By constructing a suitable Liapunov function, Gopalsamy proved that if $r \tau e^{r \tau}<1$, then the solutions of Eq. (1.2) converge to the positive equilibrium $x_{*}=\frac{1}{a_{1}+a_{2}}$.

C. Sun et al. [5] reconsidered Eq. (1.2) and showed that the positive equilibrium point is globally asymptotically stable and devote their attention to the global existence of periodic

Manuscript received April 12, 2013; revised June 20, 2013. This work is supported by the Scientific Research Center at the Erciyes University with the project codeFBA-12-4137.

F. Bozkurt is with the Department of Mathematics, ErciyesUniversity, Turkey (e-mail: fbozkurt@erciyes.edu.tr). solutions to Eq. (1.2). M. Jiang et al. [6] concerned their attention to the logistic differential equation

$$
\frac{d x(t)}{d t}=-\alpha x(t)+r \cdot x(t-\tau)\{1-x(t-\tau)\}
$$

where $\alpha$ is a known positive parameter, $r$ is an unknown parameter and $\tau>0$ is time delay. They investigated the linear stability of Eq. (1.3) by analyzing the associated characteristic transcendental equation. M. Jiang et al. reconsidered Eq. (1.3) to applied the Halanay inequality and discussed the local stability of (1.3). By constructing numerical examples they also detect nonlinear behaviors with a single parameter delay.

In this paper, we extend the delayed logistic differential equation of (1.2) by adding an extra delayed term as follows

$$
\frac{d x}{d t}=r \cdot x(t)\{1-\alpha \cdot x(t)-\beta x(t-\tau)\}+\gamma x(t-\tau)
$$

where the parameters $\alpha, \beta$ are known positive real numbers, $r, \gamma \in R-\{0\}$ and $\tau>0$ is time delay. We emphasize here the two unknown parameters $r$ and $\gamma$, which are important to determine the locally asymptotically stability of the equilibrium points of Eq. (1.4), the existence of Hopf bifurcation and the direction of the bifurcating periodic solutions. This paper is organized as follows: In Section II, using the lemma in [10] we show that the equilibrium points of Eq. (1.4) are locally asymptotically stable. Further, by constructing a suitable Liapunov function, we get that the solutions of Eq. (1.4) converge to the equilibrium points under specific conditions. In Section III, the formula are presented for determining bifurcation direction and stability of the bifurcating periodic solutions of Eq. (1.4).

\section{LOCAL STABILITY AND EXISTENCE}

With the transformation $u(t)=x(\tau t)$, we can rewrite Eq. (1.4) as the delay differential equation

$$
\frac{d u(t)}{d t}=r \tau u(t)\{1-\alpha u(t)-\beta u(t-1)\}+\gamma \tau u(t-1)
$$

This equation has two equilibrium points, which are $\bar{u}_{1}=0$ and $\bar{u}_{2}=\frac{r+\gamma}{r(\alpha+\beta)}$, where $r \neq 0$. After linearization of Eq. (2.1) at the neighborhood of $\bar{u}_{1}=0$, one gets:

$$
\frac{d u(t)}{d t}=r \tau u(t)+\gamma \tau u(t-1)
$$

The characteristic equation of Eq. (2.2) is of the form 


$$
\lambda-r \tau-\gamma \tau e^{-\lambda}=0
$$

On the other hand, after linearization of Eq. (2.1) at the neighborhood of $\bar{u}_{2}=\frac{r+\gamma}{r(\alpha+\beta)}$, one obtains:

$$
\frac{d y(t)}{d t}=-\tau\left(\alpha r \bar{u}_{2}+\gamma\right) y(t)-\tau\left(\beta r \bar{u}_{2}-\gamma\right) y(t-1),
$$

where $y(t)=u(t)-\bar{u}_{2}$. The characteristic equation of Eq. (2.4) is of the form

$$
\lambda+\tau\left(\alpha r \bar{u}_{2}+\gamma\right)+\tau\left(\beta r \bar{u}_{2}-\gamma\right) e^{-\lambda}=0
$$

Clearly, the stability of the equilibriums $\bar{u}_{1}=0$ and $\bar{u}_{2}=\frac{r+\gamma}{r(\alpha+\beta)}$ depend on the roots of the characteristic equations (2.3) and (2.5), respectively.

The following theorems are obtained by using the references [10] and [11]. Therefore, it will be omitted to the readers.

Theorem 2.1. Let $\bar{u}_{1}=0$ and $\bar{u}_{2}=\frac{r+\gamma}{r(\alpha+\beta)}$ are the equilibrium points of Eq. (2.1), where $r \neq 0$. The following statements are true.

1) Let $r<\frac{1}{\tau}$. The equilibrium point $\bar{u}_{1}=0$ of Eq. (2.1) is locally stable if $-\frac{1}{\tau} \sqrt{r^{2} \tau^{2}+\chi^{2}}<\gamma<-r \quad$,where $\chi=r \tau \tan \chi, 0<\chi<\pi$.

2) Let $\gamma>-\frac{1}{\tau}$. The equilibrium point $\bar{u}_{2}=\frac{r+\gamma}{r(\alpha+\beta)}$ of Eq.

(2.1) is locally stable if

$$
-\gamma<r<\frac{\alpha \gamma}{\beta}+\frac{1}{\beta} \sqrt{((2 \alpha+\beta) \gamma+\alpha r)^{2}\left(1+\tan ^{2} \chi\right)}
$$

where $\chi=-\frac{\tau}{\alpha+\beta}(\gamma(2 \alpha+\beta)+\alpha r) \tan \chi, 0<\chi<\pi$.

Theorem 2.2. If $\gamma^{2}<r^{2}$, then the equilibrium point $\bar{u}_{1}=0$ of Eq. (2.1) is asymptotically stable for all delay $\tau>0$.

Theorem 2.3. If $\beta>\alpha$ and $r \in\left(\frac{4 \alpha \gamma-\sqrt{\Delta}}{2(\beta-\alpha)}, \frac{4 \alpha \gamma+\sqrt{\Delta}}{2(\beta-\alpha)}\right)$, where $\Delta=4 \gamma^{2}(\alpha+\beta)^{2}$, then the equilibrium point $\bar{u}_{2}=\frac{r+\gamma}{r(\alpha+\beta)}$ of Eq. (2.1), where $r \neq 0$ is asymptotically stable for all delay $\tau>0$.

Theorem 2.4. Let $\gamma<0, r>0$. Suppose for $\tau>0$ that $0<x(t)<\sqrt{2 r+1} \cdot x(t)<x(t-\tau)$. Then the equilibrium point $\bar{u}_{1}=0$ of Eq. (1.4) is globally asymptotically stable.

Theorem 2.5. Let $0<r<-\frac{(\alpha+\beta)+2 \alpha \gamma}{2(2 \alpha+\beta)}<-\gamma \quad$ and $\gamma<-\frac{\alpha+\beta}{2 \alpha}$. Suppose that $0<x(t)<x(t-\tau)$ for $\tau>0$. Then the equilibrium point $\bar{u}_{2}=\frac{r+\gamma}{r(\alpha+\beta)}$ of Eq. (1.4) is globally asymptotically stable.

Theorem 2.6. Let $\quad \gamma=-\frac{1}{\tau} \sqrt{r^{2} \tau^{2}+\chi^{2}} \quad$,where $\chi=r \tau \tan \chi$ and $0<\chi<\pi$. Then there is a Hopf bifurcation from the equilibrium $\bar{u}_{1}=0$ to a periodic orbit.

It can be similarly proven that when $r$ passes the critical point

$$
r_{*}=\frac{\alpha \gamma}{\beta}+\frac{1}{\beta} \sqrt{\left((2 \alpha+\beta) \gamma+\alpha r_{*}\right)^{2}\left(1+\tan ^{2} \chi\right)},
$$

where $\chi=-\frac{\tau}{\alpha+\beta}\left(\gamma(2 \alpha+\beta)+\alpha r_{*}\right) \tan \chi \quad, \quad 0<\chi<\pi$, there is a Hopf bifurcation from the equilibrium $\bar{u}_{2}=\frac{\gamma+r}{r(\alpha+\beta)}$ to a periodic orbit.

\section{StABility AND Direction OF THE BIfURCATING PERIODIC SOLUTIONS}

Let $\gamma=\gamma_{*}+v$, where $v \in R$. In this case $v=0$ is one Hopfbifurcation value for Eq. (2.1). For $\mu \in C$, let

$$
L_{v} \mu=r \tau \mu(0)+\left(\gamma_{*}+v\right) \cdot \tau \mu(-1)
$$

and

$$
F(\nu, \mu)=-\alpha r \tau \mu^{2}(0)-\beta \tau r \mu(0) \mu(-1)
$$

By the Riesz representation theorem, there exists a function $\eta(\theta, v)$ of bound variation for $\theta \in[-1,0]$, such that

$$
L_{v} \mu=\int_{-1}^{\theta} d \eta(\theta, v) \mu(\theta) \text { for } \mu \in C
$$

In fact, we can choose

$$
\eta(\theta, v)=r \tau \delta(\theta)+\left(\gamma_{*}+v\right) \cdot \tau \delta(\theta+1)
$$

where $\theta \in[-1,0]$ and $\delta$ is the Dirac delta function. For $\mu \in C^{1}([-1,0], R)$, define

$$
A(v) \mu=\left\{\begin{array}{cr}
\frac{d \mu(\theta)}{d \theta}, & -1 \leq \theta<0 \\
\int_{-1}^{0} \mathrm{~d} \eta(\xi, v) \mu(\xi), & \theta=0,
\end{array}\right.
$$

and

$$
B(v) \mu=\left\{\begin{array}{cr}
0, & -1 \leq \theta<0 \\
\mathrm{~F}(v, \mu), & \theta=0 .
\end{array}\right.
$$

Hence, (2.1) can be rewritten as 


$$
\dot{u}_{t}=A(v) u_{t}+B(v) u_{t}
$$

where $u_{t}(\theta)=u(t+\theta)$ for $\theta \in[-1,0]$.

For $\psi \in C^{1}[-1,0]$, we define the adjoint operator $A^{*}$ of $A$ as

$$
A^{*}(v) \psi=\left\{\begin{array}{cl}
-\frac{d \psi(\xi)}{d \xi}, & 0<\xi \leq 1 \\
\int_{-1}^{0} \mathrm{~d} \eta(t, v) \mu(-t), & \xi=0
\end{array}\right.
$$

and a bilinear inner product as

$$
\langle\psi, \mu\rangle=\bar{\psi}(0) \mu(0)-\int_{-1}^{0} \int_{s=0}^{\theta} \bar{\psi}(s-\theta) d \eta(\theta, 0) \mu(s) d s
$$

To determine the Poincare normal form of $A$, we need to calculate the eigenvector $q$ of $A$ belonging to the eigenvalue $\mathrm{i} \omega_{0}$ and eigenvector $q^{*}$ of $A^{*}$ belonging to the eigenvalue $i \omega_{0}$. It can be verified that $q(\theta)=e^{i \omega_{0} \theta}$ is an eigenvector of $\mathrm{A}(0)$ corresponding to $i \omega_{0}$, and $q^{*}(s)=D e^{i \omega_{0} s}$ is an eigenvector of $A^{*}(0)$ with respect to the eigenvalue $i \omega_{0}$. Furthermore, by (3.8), we have $A^{*} q^{*}(\theta)=-i \omega_{0} q^{*}(\theta)$.

We normalize $q$ and $\mathrm{q}^{*}$ by the condition $\left\langle q^{*}, q\right\rangle=1$ to determine the value of D. From (3.9), we have

$$
\begin{aligned}
\left\langle q^{*}, q\right\rangle & =\bar{D}-\int_{-1}^{0} \int_{s=0}^{\theta} \bar{q}^{*}(s-\theta) d \eta(\theta, 0) \mu(s) d s \\
& =\overline{\mathrm{D}}\left(1+\gamma_{*} \pi \mathrm{e}^{-\mathrm{i} \omega_{0}}\right)=1
\end{aligned}
$$

Hence

$$
q^{*}(\theta)=\frac{1}{1+\gamma_{*} \tau e^{i \omega_{0}}} e^{i \omega_{0} \theta}, \quad 0 \leq \theta \leq 1
$$

Similarly, we have $\left\langle q^{*}, \bar{q}\right\rangle=0$.

To describe the center manifold $C_{0}$ at $v=0$, we want to compute the coordinates. For each $u \in \operatorname{Dom}(A)$, we associate the pair $(z, s)$, where $z=\left\langle q^{*}, u_{t}\right\rangle$ and

$$
s(t, \theta)=u_{t}(\theta)-z q(\theta)-\bar{z} \bar{q}(\theta)=u_{t}(\theta)-2 \operatorname{Re}\{z q(\theta)\}
$$

On the center manifold $C_{0}$, we have $s(t, \theta)=s(z(t), \bar{z}(t), \theta)$ , where

$$
s(z, \bar{z}, \theta)=s_{20}(\theta) \frac{z^{2}}{2}+s_{11}(\theta) z \bar{z}+s_{02}(\theta) \frac{\bar{z}^{2}}{2}+s_{30}(\theta) \frac{z^{3}}{6}+\ldots
$$

Here are $z$ and $\bar{z}$ local coordinates for center manifold $C_{0}$ in the direction of $q$ and $q^{*}$. Note that $s(t, \theta)$ is real, if $u_{t}$ is real. We only deal with real solutions. It is easy to show that $\left\langle q^{*}, s\right\rangle=0$. For solutions $u_{t} \in C_{0}$ of Eq. (2.1) we have

$$
\left\langle q^{*}, \dot{u}_{t}\right\rangle=\left\langle q^{*}, A u_{t}+B u_{t}\right\rangle
$$

Since $v=0$, we have

$$
\begin{aligned}
\dot{z}_{t} & =\left\langle q^{*}, \dot{u}_{t}\right\rangle=\left\langle q^{*}, A u_{t}+B u_{t}\right\rangle \\
& =i \omega_{0} z_{t}+\bar{q}^{*}(0) f(z, \bar{z}) .
\end{aligned}
$$

We rewrite the above differential equation as

$$
\dot{z}(t)=i \omega_{0} z+\bar{q}^{*}(0) f(z, \bar{z})=i \omega_{0} z+g(z, \bar{z})
$$

where

$$
g(z, \bar{z})=\bar{q}^{*}(0) f(z, \bar{z})=g_{20} \frac{z^{2}}{2}+g_{11} \bar{z}+g_{02} \frac{\bar{z}^{2}}{2}+g_{30} \frac{z^{3}}{6}+\ldots
$$

From (3.11), we have

$$
\begin{gathered}
\mathrm{u}_{\mathrm{t}}(\theta)=\mathrm{s}(\mathrm{t}, \theta)+2 \operatorname{Re}\{\mathrm{zq}(\theta)\}=\mathrm{s}_{20}(\theta) \frac{\mathrm{z}^{2}}{2}+ \\
\mathrm{s}_{11}(\theta) \mathrm{z} \overline{\mathrm{Z}}+\mathrm{s}_{02}(\theta) \frac{\overline{\mathrm{z}}^{2}}{2}+\mathrm{e}^{\mathrm{i} \omega_{0} \theta} \mathrm{z}+\mathrm{e}^{-\mathrm{i} \omega_{0} \theta} \overline{\mathrm{z}}+\ldots
\end{gathered}
$$

Using (3.2) and (3.15) in (3.14), we get

$$
\begin{aligned}
g(z, \bar{z}) & =\bar{q}^{*}(0) f(z, \bar{z}) \\
& =\bar{q}^{*}(0) F\left(0, u_{t}\right) \\
& =-\overline{\operatorname{Dr}} \tau\left[\alpha u_{t}^{2}(0)+\beta u_{t}(0) u_{t}(-1)\right]
\end{aligned}
$$

Comparing the coefficientswith (3.14) and (3.16), we have

$$
\begin{gathered}
\mathrm{g}_{20}=-2 \overline{\mathrm{D}} \tau\left(\alpha+\beta \mathrm{e}^{-\mathrm{i} \omega_{0}}\right) \\
\mathrm{g}_{11}=-2 \overline{\mathrm{D}} \tau\left(\alpha+\beta \cos \omega_{0}\right) \\
\mathrm{g}_{02}=-2 \overline{\mathrm{D}} \tau\left(\alpha+\beta \mathrm{e}^{\mathrm{i} \omega_{0}}\right) \\
\mathrm{g}_{21}=-\overline{\mathrm{D}} \tau\left[\left(2 \alpha\left(\mathrm{s}_{20}(0)+2 \mathrm{~s}_{11}(0)\right)+\right.\right. \\
\left.\beta\left(\mathrm{e}^{\mathrm{i} \omega_{0}} \mathrm{~s}_{20}(0)+\mathrm{s}_{20}(-1)+2 \mathrm{~s}_{11}(-1)+2 \mathrm{e}^{-\mathrm{i} \omega_{0}} \mathrm{~s}_{11}(0)\right)\right]
\end{gathered}
$$

Since $s_{20}$ and $s_{11}$ are unknown in $g_{21}$, we want to compute and determine them.From (3.11) and (3.13), we obtain

$$
\begin{gathered}
\dot{s}=\dot{u}_{t}-q \dot{z}-\bar{q} \dot{z} \\
=A w-2 \operatorname{Re}\left\{\bar{q}^{*} f(z, \bar{z}) q\right\}+B u_{t} .
\end{gathered}
$$

So, we have by the definition of B in Eq. $(3,6)$,

$$
\dot{s}=\dot{u}_{t}-q \dot{\bar{z}}-\bar{q} \dot{\bar{z}}=\left\{\begin{array}{lr}
\text { As }-2 \operatorname{Re}\left\{\bar{q}^{*} f(z, \bar{z}) q(\theta)\right\}, & -1 \leq \theta<0 \\
\text { As }-2 \operatorname{Re}\left\{\bar{q}^{*} f(z, \bar{z}) q\right\}+f(z, \bar{z}) & \theta=0 .
\end{array}\right.
$$

We rewrite (3.17) as 


$$
\dot{s}(t)=A s+K(z, \bar{z}, \theta)
$$

where

$$
K(z, \bar{z}, \theta)=K_{20}(\theta) \frac{z^{2}}{2}+K_{11}(\theta) z \bar{z}+K_{02}(\theta) \frac{\bar{z}^{2}}{2}+\ldots
$$

Substituting the corresponding series into (3.17) and comparing the coefficients, we obtain

$$
\left(A-2 i \omega_{0}\right) s_{20}(\theta)=-K_{20}(\theta), \operatorname{As}_{11}(\theta)=-K_{11}(\theta), \ldots(3.20)
$$

Using (3.19) in (3.17), we have

$$
K_{20}(\theta) \frac{z^{2}}{2}+K_{11}(\theta) z \bar{z}+K_{02}(\theta) \frac{\bar{z}^{2}}{2}+\bar{q}^{*}(0) f(z, \bar{z}) q+q^{*}(0) \overline{f(z, \bar{z}) \bar{q}}+\ldots=0
$$

which can also be written as

$$
K_{20}(\theta) \frac{z^{2}}{2}+K_{11}(\theta) z \bar{z}+K_{02}(\theta) \frac{\bar{z}^{2}}{2}+\ldots+g(z, \bar{z}) q+\overline{g(z, \bar{z}) q}=0
$$

Comparing these coefficients with those of (3.14), we get

$$
\left\{\begin{array}{l}
K_{20}(\theta)=-g_{20} q(\theta)-\overline{g_{02}} \cdot \bar{q}(\theta) \\
K_{11}(\theta)=-g_{11} q(\theta)-\overline{g_{11}} \bar{q}(\theta) \\
K_{02}(\theta)=-g_{02} q(\theta)-\overline{g_{20}} \bar{q}(\theta) \\
\cdots
\end{array}\right.
$$

From (3.17), (3.21) and by the definition of A, that we have

$$
\dot{s}_{20}(\theta)=2 i \omega_{0} s_{20}+g_{20} q(\theta)+\overline{g_{02}} \bar{q}(\theta)
$$

and

$$
\dot{s}_{11}(\theta)=g_{11} q(\theta)+\overline{g_{11}} \bar{q}(\theta)
$$

Since $q(\theta)=e^{i \omega_{0} \theta}$, solving (3.22) and (3.23), we obtain

$$
s_{20}(\theta)=i \frac{g_{20}}{\omega_{0}} e^{i \omega_{0} \theta}+i \overline{g_{02}} \frac{\omega_{0}}{3 \omega_{0} \theta}+U_{1} e^{2 i \omega_{0} \theta}
$$

and

$$
s_{11}(\theta)=-i \frac{g_{11}}{\omega_{0}} e^{i \omega_{0} \theta}+i \frac{\overline{g_{11}}}{\omega_{0}} e^{-i \omega_{0} \theta}+U_{2}
$$

where $U_{1}$ and $U_{2}$ are constants. From the definition of $A$, (3.17) and (3.20), we can write

$$
\left\{\begin{array}{l}
K_{20}(0)=-g_{20}-\overline{g_{02}}-2 r \tau\left(\alpha+\beta e^{-i \omega_{0}}\right)=-A s_{20}(0)+2 i \omega_{0} s_{20}(0), \\
K_{11}(0)=-g_{11}-\overline{g_{11}}-2 r \tau\left(\alpha+\beta \cos \omega_{0}\right)=-A s_{11}(0), \\
\ldots
\end{array}\right.
$$

From the first equation of system (3.26), we obtain

$$
-g_{20}-\overline{g_{02}}-2 r \tau\left(\alpha+\beta e^{-i \omega_{0}}\right)=-r \tau s_{20}(0)-\gamma_{*} \tau s_{20}(-1)+2 i \omega_{0} s_{20}(0)
$$

From this, we get

$$
U_{1}=\frac{i g_{20}\left(\frac{r \tau+\gamma_{*} \tau e^{-i \omega_{0}}}{\omega_{0}}-i\right)+i \bar{g}_{02}\left(\frac{r \tau+\gamma_{*} i e^{i \omega_{0}}}{3 \omega_{0}}+\frac{i}{3}\right)-2 r \tau\left(\alpha+\beta e^{-i \omega_{0}}\right)}{2 i \omega_{0}-r \tau-\gamma_{*} \tau e^{-2 i \omega_{0}}}
$$

By the second equation of system (3.26), we have

$$
-g_{11}-\overline{g_{11}}-\left(\alpha+\beta \cos \omega_{0}\right)=-r \tau s_{11}(0)-\gamma_{*} \tau s_{11}(-1)
$$

Computing it, we obtain

$U_{2}=\frac{i g_{11}\left(\frac{r \tau+\gamma_{*} \tau e^{-i \omega_{0}}}{\omega_{0}}-i\right)-i \bar{g}_{11}\left(\frac{r \tau+\gamma_{*} \tau e^{i \omega_{0}}}{\omega_{0}}+i\right)+2 r \tau\left(\alpha+\beta \cos \omega_{0}\right)}{\tau\left(r+\gamma_{*}\right)}$

Finally, from (3.24), (3.25), (3.27) and (3.28), we can see that each $g_{i j}(\mathrm{i}, \mathrm{j}=0,1,2, \ldots$.$) is determined by the parameters$ and delays in Eq. (2.1). Thus, we can compute the following values

$$
\left\{\begin{array}{l}
c_{1}(0)=\frac{i}{2 \omega_{0}}\left(g_{20} g_{11}-2\left|g_{11}\right|^{2}-\frac{1}{3}\left|g_{02}\right|^{2}\right)+\frac{g_{21}}{2}, \\
\mu_{2}=-\frac{\operatorname{Re}\left(c_{1}(0)\right)}{\operatorname{Re}\left(\lambda^{\prime}\left(\gamma_{*}\right)\right)} \\
\beta_{2}=2 \operatorname{Re}\left(c_{1}(0)\right) .
\end{array}\right.
$$

A general result for the direction and stability of Hopf bifurcation ([12], Chapter 1, Section IV), implies that the direction of the Hopf bifurcation is determined by the sign of $\mu_{2}$ and the stability of bifurcating periodic solutions by the sign of $\beta_{2}$, respectively. In this case, if $\mu_{2}>0(<0)$, then the Hopf bifurcation is supercritical (subcritical) and if $\beta_{2}<0$ $(>0)$ the bifurcating periodic solution are orbitally stable (unstable).

\section{EXAMPLE}

1) From Theorem 2.6, using $\tau=0.5, r=-4.71238898025$, $\alpha=0.5, \quad \beta=0.8999999, \quad \gamma=-6.66432399526318$ and $x(0)=0.8001$, the graph of the first 50 iterations of Eq. (1.4) is given in Fig. 1 (a) and Fig. 1 (b). These figures show us that there is a Hopf bifurcation from the equilibrium point $\bar{u}_{1}=0$ of Eq. (2.1) to a periodic orbit.

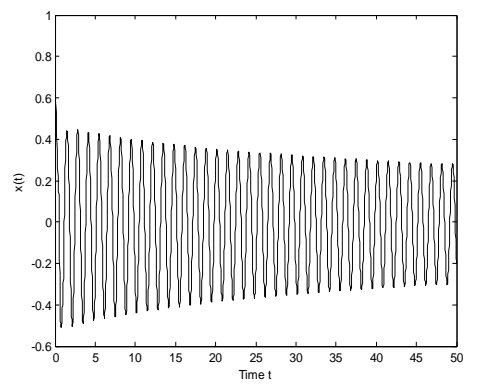

Fig. 1 (a) 


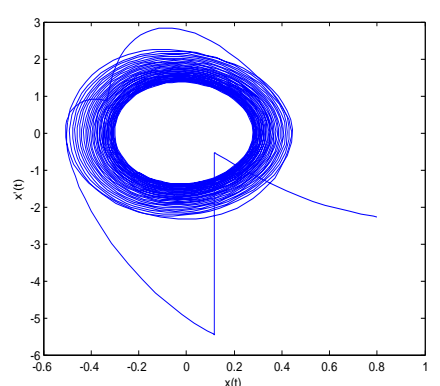

Fig. 1 (b)

2) Using $\tau=0.5, \quad r=1.5707963275, \alpha=0.0005$, $\beta=0.0003, \gamma=-2.221441470076$ and $x(0)=0.8001$, the graph of the first 50 iterations of Eq. (1.4) is given in Fig. 2 (a) and Fig. 2 (b). We obtain that there is a Hopf bifurcation from the equilibrium point $\bar{u}_{2}$ of Eq. (2.1) to a periodic orbit.

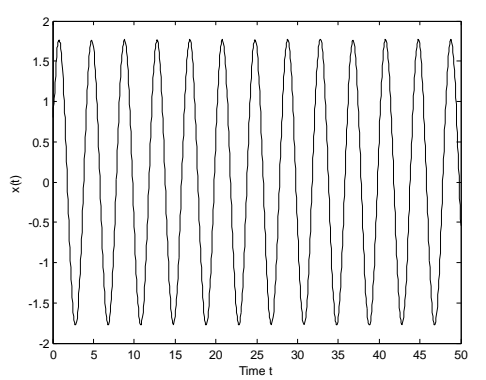

Fig. 2 (a)

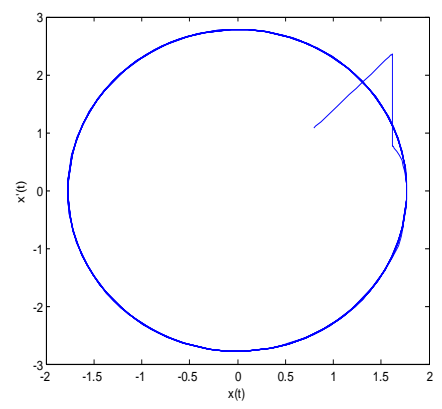

Fig. 2(b)

\section{DISCUSSION}

It can be shown that if we use the parameters in Example 1 ), to get the values of (3.28), then we obtain $C_{1}(0)=-0.64676-0.49156 i \quad, \quad \mu_{2}=-5.3852 \quad$ and $\beta_{2}=-1.29352$. In this case, Fig. 1 (a) and Fig. 1 (b) show us that the Hopf bifurcation is subcritical and thatbifurcating periodic solution areorbitallystable.

Similarly, in Example 2), computations give that $C_{1}(0)=0.00018155-0.00055611 i, \quad \mu_{2}=0.00059634$ and $\beta_{2}=0.0003631$, which show that in Fig. 2(a) and Fig. 2 (b), we have a supercritical Hopf bifurcation. Therefore,these bifurcating periodic solutions are orbitally unstable.

\section{ACKNOWLEDGMENT}

This work is supported by the Scientific Research Center at the Erciyes University with the project codeFBA-12-4137.

\section{REFERENCES}

[1] G. E. Hutchinson, Circular causal systems in ecology, Ann. N. Y. Acad. Sci., vol. 50, pp. 221- 246, 1948.

[2] K. Gopalsamy, Stability and oscillations in delay differential equations of a population dynamics, Dodrecht: Kluwer Academic Press, 1992.

[3] Y. Kuang, Delay Differential Equations with Applications in Population Dynamics, Academic Press, Boston 1993.

[4] M. Jiang et al., "Nonlinear behavior of the parameterized logistic differential systems," Applied Mathematics and Computation, vol. 89, pp. 1694-1704, 2007.

[5] C. Sun, M. Han, and Y. Lin, "Analysis of stability and Hopf bifurcation for delayed logistic equation," Chaos, Solutions and Fractals, vol. 31, pp. 672-682, 2007.

[6] M. Yiang et al., "Stability, bifurcation and a new chaos in the logistic differential equation with delay," Physics Letters A, vol. 350, pp. 221-227, 2006.

[7] K. Gopalsamy, "On the global atrractivity in a generalized delay-logistic differential equation," in Proc. Camb. Philos. Soc., vol. 100, pp. 183-192, 1986.

[8] H. Betz, P. B. Burcham, and G. M. Ewig, Differential equation with application, Harper and Brothers, New York, 1954.

[9] J. Uchanski, Classical Mathematical Ecology, PWN, Warsaw, 1992.

[10] J. Hale and S. Verduyn Lunel, Introduction to Functional Differential equations, Springer, New York, 1993.

[11] S. Ruan and J. Wei, "On the zeros of transcendental functions with applications to stability of delay differential equations," Dynam Cont. Discr.Impul.Sys. Series A: Math. Anal., vol. 1, pp. 863-874, 2003.

[12] B. Hassard, D. Kazarino, and Y. Wan, Theory and applications of Hopfbifurcation, Cambridge University Press, 1981.

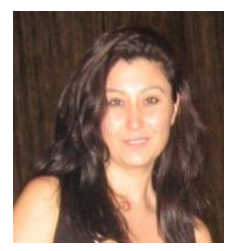

Fatma Bozkurt was born in Germany in 1979. Since 1994 she lives in Turkey. She studied Mathematics at the Erciyes University (1998-2002), and has two MSci, which one is about Applied Mathematics( "The periodic character of the solutions of some difference equation", Erciyes University, Turkey, 2002-2005 and the other about Mathematics Education (Hacettepe University, Turkey, 2003-2005). Her PhD thesis is about the population dynamics of single species ("Stability Analysis for Differential and Difference Equations in Population Models, Erciyes University, Turkey, 2006-2010).

She worked from 2005 until 2010 as Research Assistant at the Erciyes University and got the Assis. Prof. Dr. title in 2010. Since 2010 she is working as Assis. Prof. Dr. at the Erciyes University in Kayseri (Turkey). Her research fields are about the dynamics of species and especially about modeling and analyzing the brain tumor GBM.

She is head of some projects about modeling GBM tumor and the epidemic disease HIV/AIDS(FBA-12-4137, FBY-12-3993) and is Researcher at the project FBA-11-3597. 